\title{
Graphene-ribbon Model for Nano-meter Size Magnetic Recording Bit
}

\author{
Norio Ota \\ Graduate School of Pure and Applied Sciences, University of Tsukuba, 1-1-1 Tenoudai Tsukuba-city 305-8571, Japan
}

\begin{abstract}
Magnetic graphene-ribbon is a candidate for realizing future ultra high density 100 tera bit/inch ${ }^{2}$ class data storage media. Multiple spin state analysis was done based on the density functional theory. A typical model has a super-cell $\left[\mathrm{C}_{80} \mathrm{H}_{7}\right]$ which keeping bare (radical) carbons on one side zigzag edge, whereas mono hydrogenated carbons on another side. Optimizing atomic configuration, self-consistent calculation demonstrated that a total energy of the highest spin state is more stable than that of the lower one, which origin comes from the exchange coupling between carbons. This analysis suggested a capability of designing magnetic data track utilizing such asymmetric graphene ribbon. In order to increase areal magnetization density, bi-layer and quadric layer model were analyzed. Detailed calculation resulted that also the highest spin state is the most stable one. Increasing layer numbers is an effective way to enhance strong magnetism.
\end{abstract}

Key words: magnetic recording, graphene, thin film, first principles calculation, density functional theory

\section{Introduction}

Current magnetic data storage ${ }^{1)-2}$ has a density around 1 tera-bit/inch ${ }^{2}$ with $10 \mathrm{~nm}$ length, $25 \mathrm{~nm}$ width magnetic bit. Ultimate areal density will be $10^{4}$ tera-bit/inch $^{2}$ utilizing atomic scale materials ${ }^{3)}$ as illustrated in Fig.1. There is a missing link between those two density regions. In order to link those two, one promising candidate is a ferromagnetic molecule dot array having a typical areal bit size of $1 \mathrm{~nm}$ by 2.5 nm. Recently, carbon based room-temperature ferromagnetic materials are experimentally reported ${ }^{4)-9)}$. They are graphite and graphene like materials. From a theoretical view point, Kusakabe and Maruyama ${ }^{10)-11)}$ proposed an asymmetric graphene-ribbon model with two hydrogen modified (dihydrogenated) zigzag edge carbon showing ferromagnetic behavior. Our previous papers ${ }^{12)-14)}$ have reported multiple spin state analysis of graphene like molecules modified zigzag edge carbon by several atomic species. In case of bare (radical) carbon, and dihydrogenated carbon edge molecules, the highest spin state exhibits the most stable spin state, which suggest a capability of strong magnetism. Those carbon based material is very attractive for a future ultra high density (over 100 tera-bit/inch ${ }^{2}$ ) magnetic recording media. Especially, graphene-ribbon has a nano-meter width and long straight line looks like a recording track. Here, magnetic properties of chemically edge modified asymmetric graphene-ribbon and geometrical optimization are analyzed based on a first principles theory for designing a future ultra high density magnetic storage.

\section{Designing ferromagnetic graphene-ribbon}

Ohldag et al.9) recently found that proton ion implanted graphite exhibits strong surface magnetization at a room-temperature. In order to explain such remarkable experiment, we proposed an shadow effect model ${ }^{13)}$, which means that proton are partially modified zigzag edge carbon by some masking effect resulting strong magnetism. Such a model is simply illustrated in Fig. 2 with adding a mask layer. In an industrial view point, such a mask size and position will be controlled very precisely by some proper resist dot array and/or self organized thin film array. Based on such mask model on a graphene-ribbon, typical calculation example is shown in Fig.3. In order to have a discrete magnetic bit on one straight graphene-ribbon, three carbon edges on one side zigzag edge are bare, whereas another side zigzag edge are all mono hydrogenated as marked by red square. Between two magnetic bits, there is a zero magnetization buffer part (blue marked square) with mono hydrogenated edges on both upper and lower zigzag edges. One periodic super-cell for an infinite length analysis is shown in black marked square as $\left[\mathrm{C}_{80} \mathrm{H}_{7}\right]$. There are several spin states for the same super-cell. This paper studies which multiple spin state will have the lowest total energy showing a stable magnetism. If high spin state is favorable, we can expect strong magnetic behavior and application to magnetic data storage. This paper also extends to bi-layer and quadric layer ribbon model in order to increase an areal magnetization density per bit on a recording track.

\section{Calculation Method}

We have to obtain the (i) spin density, (ii) total energy, and (iii) optimized atom configuration depending on a respective given spin state $\mathrm{Sz}$ to clarify magnetism. Density functional theory (DFT) ${ }^{15)}$-16) based generalized gradient approximation (GGA-PBEPBE) ${ }^{17)}$ was applied utilizing Gaussian03 package ${ }^{18)}$ with an atomic orbital 6-31G basis set ${ }^{19)}$. We selected the same GGA-PBEPBE 
potential with previously discussed graphene molecule models 12)-14). In this paper, total charge of model super-cell is set to be completely zero. In this unrestricted DFT calculation, $\mathrm{S}(\mathrm{S}+1)$ value is obtained to check a degree of spin contamination. Spin contamination was so small for every case in this paper. For example, in case of quadric layer model with $\mathrm{Sz}=12 / 2, \quad \mathrm{~S}(\mathrm{~S}+1) \quad$ was 42.2 comparing with no contamination value $\mathrm{Sz}(\mathrm{Sz}+1)=42.0$, which means very small spin contamination. Inside of a super-cell, three dimensional DFT calculation was done. One dimensional periodic boundary condition was applied to realize an unlimited length graphene-ribbon.

Self-consistent energy, atomic configuration and spin density calculations are repeated until to meet convergence criteria. The required convergence on the root mean square density matrix was less than $10 \mathrm{E}-8$ within 128 cycle.

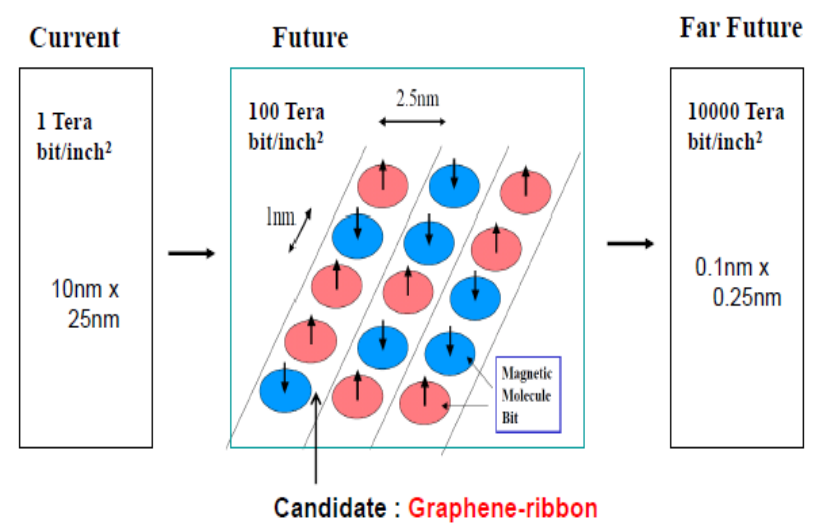

Fig.1 Future ultra-high density 100 tera-bit/inch ${ }^{2}$ magnetic data storage with bit size of $1 \mathrm{~nm}$ by $2.5 \mathrm{~nm}$, which may link current and far future density region. Graphene-ribbon is a candidate for such application.

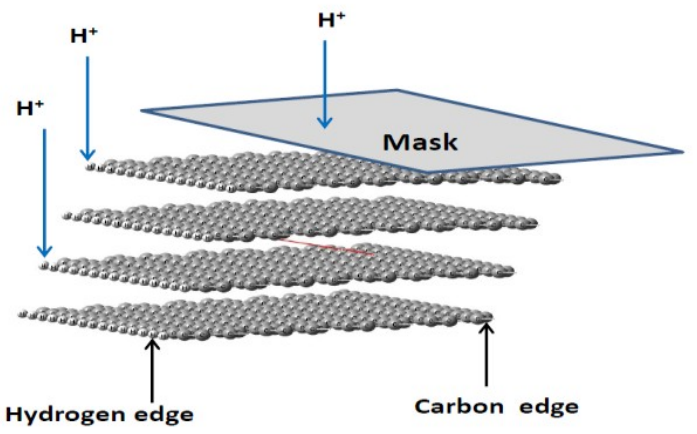

Fig.2 Proton irradiated graphite model. By a shadow effect using some tiny mask, left side carbon edge is mono-hydrogenated, whereas right side one remains as bare (radical) carbon. Such chemical modification causes strong magnetism.

\section{4, Monolayer ribbon}

Typical monolayer model is shown in Fig.3. Each super-cell has limited numbers of unpaired electrons, which enable allowable numbers of multiple spin states. Optimized atomic configuration result a flat and straight ribbon. Some theoretical papers suggested that narrow width unsaturated ribbon less than $1.5 \mathrm{~nm}$ may show some twisting and uniform curving ${ }^{22) 23)}$. However, our model has sufficient large width (1.8nm) not occurring such irregularity. By an insertion of saturated buffer part shown in Fig.3 (blue area), ripples and warpings ${ }^{22}$ are relaxed. In a super cell $\left[\mathrm{C}_{80} \mathrm{H}_{7}\right]$, there are three unpaired electrons which bring two spin state like $\mathrm{Sz}=3 / 2$ and $1 / 2$. These $\mathrm{Sz}$ values are installed as spin parameter to start DFT calculations. Repeating DFT self consistent calculation, final obtained spin density maps are shown in Fig.4, comparing (a) $\mathrm{Sz}=3 / 2$ and (b) $1 / 2$, where up-spins are indicated in red (dark gray), and down-spins in blue (light gray) at a $0.001 \mathrm{e}^{3} \mathrm{~A}^{3}$ contour spin density surface. In case of (a) $\mathrm{Sz}=3 / 2$ (high spin state), we found that up and down spin clouds were very regularly aligned one by one. It should be noted that summed atomic spin density of a bare carbon was 1.36 $\mathrm{M}_{\mathrm{B}}$ and a nearest carbon $-0.27 \mathrm{M}_{\mathrm{B}}$ (negative sign). In contrast, in case of (b) $\mathrm{Sz}=1 / 2$ (low spin state) a very complex spin structure appeared like up-up and down-down spin pairs. Exchange coupling between up-up (also down-down) brought about a local exchange energy increase, and finally elevated the total energy. Exact total super-cell energy of high spin state $(\mathrm{Sz}=3 / 2)$ is $13.2 \mathrm{kcal} / \mathrm{mol}$ lower than that of low spin state $(\mathrm{Sz}=1 / 2)$. It should be noted that high spin state is more stable than low spin state.

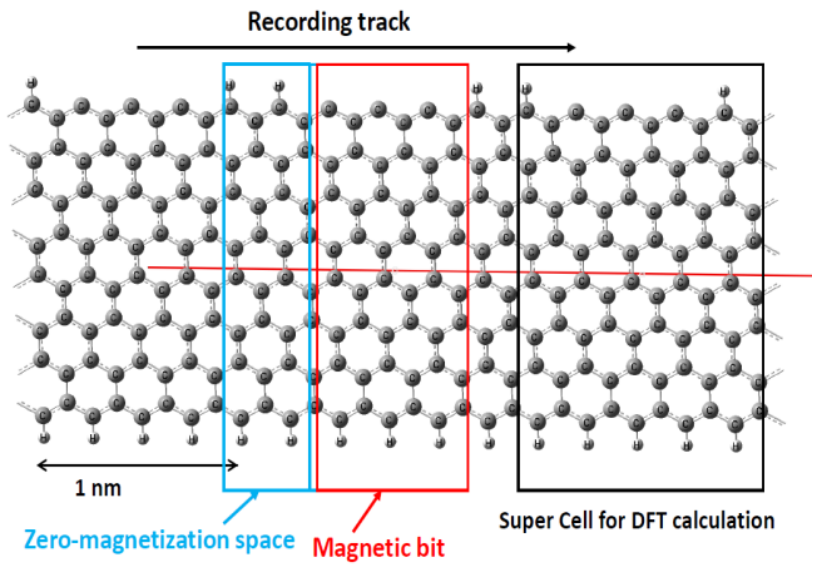

Fig.3 Design of a magnetic bit on a single layer graphene-ribbon (red marked square). Blue marked area is a buffer to distinct bit to bit. Super-cell for an infinite length ribbon calculation is shown in black mark as $\left[\mathrm{C}_{80} \mathrm{H}_{7}\right]$.

\section{Multilayer ribbon}

In order to increase the areal saturation magnetization per bit, increasing layer numbers is an effective way. Of 
course, we need to check magnetic stability and atomic configuration. Here, bi-layer and quadric layer magnetic graphene-ribbon are studied. Typical bi-layer model is shown in Fig.5. (a) $\mathrm{S}$

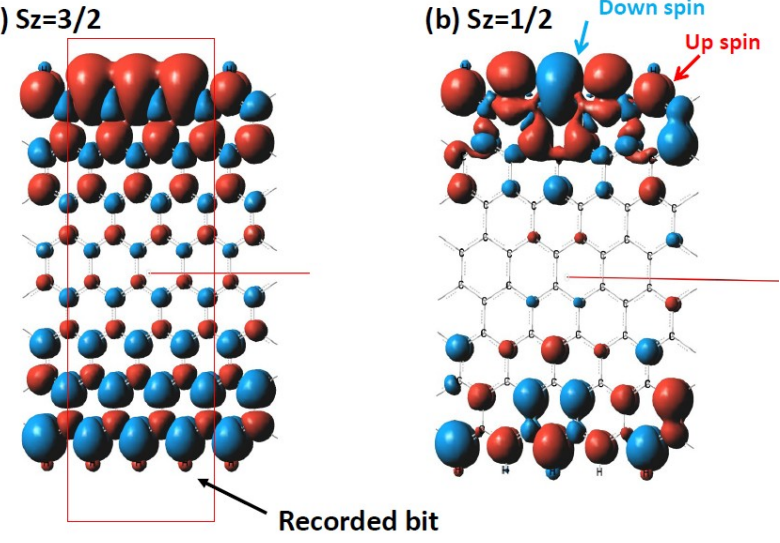

Fig. 4 Spin density map of $\mathrm{Sz}=3 / 2$ in (a) and $\mathrm{Sz}=1 / 2$ in (b) in a super-cell $\left[\mathrm{C}_{80} \mathrm{H}_{7}\right]$, where up-spins are indicated in red (dark gray), and down-spins in blue (light gray). Total energy of high spin state (a) is $13.5 \mathrm{kcal} / \mathrm{mol}$ lower than that of low spin state (b). High spin state is stable than lower one, which suggest a strong magnetism.

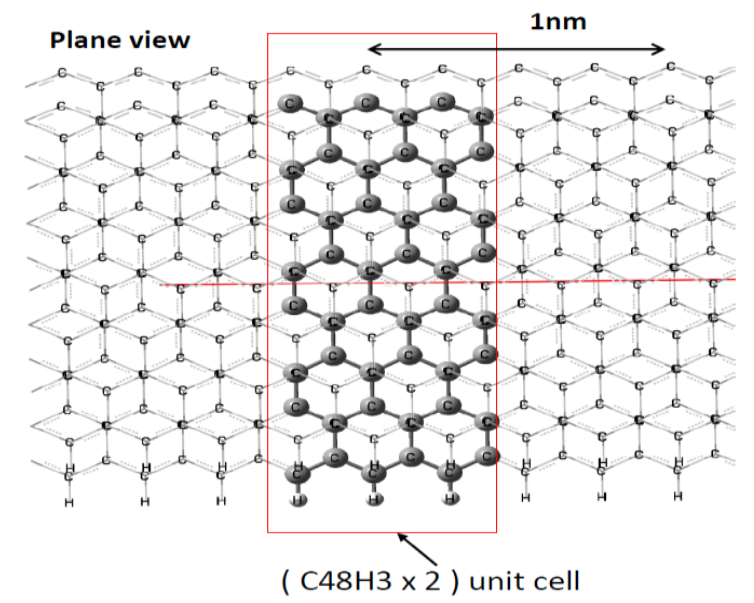

Fig.5 Plane view of a bi-layer magnetic graphene-ribbon with the AC stacked structure having a super-cell $\left[\left(\mathrm{C}_{48} \mathrm{H}_{3}\right) \times 2\right]$. Red squared area show a super-cell, where ball symbolized carbon and small ball hydrogen demonstrate a back (second) layer.

Super-cell is $\left[\mathrm{C}_{48} \mathrm{H}_{3} \times 2\right.$ ], which means $\mathrm{C}_{48} \mathrm{H}_{3}$ monolayer cell overlaps one by one to construct bi-layer. Red squared area show a super-cell, where ball carbon and hydrogen demonstrate a back (second) layer. Zheng et al ${ }^{20)}$ experimentally observed bi-layer graphene zigzag edge structure by means of atomically resolved high-resolution TEM. They indicated that bi-layer graphene is stable at a room-temperature with the AA stacking, mostly showing closed (rolled) ends but partially having open zigzag edges. Depending on such observation, our initial atomic configuration was the AA stacking bi-layer graphene-ribbon as illustrated in Fig.6 (a), which is a side view with open edges and layer to layer distance of $0.334 \mathrm{~nm}$ with a typical bulk graphite value. Executing DFT calculation, dynamic forces between atoms gradually enforce two layers moving and sliding each other, and after 95 times self consistent calculation cycles, it finally converged as the $\mathrm{AC}$ stacking structure 21) with a distance of $0.304 \mathrm{~nm}$ as shown in (b). Additionally we could find that bare carbon edges (upper end in (b)) become close together. Comparing with an experimental result ${ }^{20}$ ) after 2000 degree $\mathrm{C}$ high temperature annealing, our DFT calculation is limited to quantum force balance at a zero temperature, and may not to have enough force to grow up to AA stacking and/or AB stacking .

In our bi-layer model, there are three multiple spin state capability as $\mathrm{Sz}=6 / 2,2 / 2$ and 0/2. Result is shown in Fig. 7 as spin density map (plane view). In case of the highest spin state (a) $\mathrm{Sz}=6 / 2$, up and down spin clouds were very regularly aligned one by one. In contrast, in case of (b) $\mathrm{Sz}=2 / 2$, a very complex spin structure appeared which increases exchange energy and total energy by $1.6 \mathrm{kcal} / \mathrm{mol}$ per super-cell. Comparing a single layer case, this energy difference is small. Capable reason is a complex atomic structure of bare carbon edges becoming close together. Up-spin clouds overlaps each together and elevate an exchange energy between two layers. The lowest spin state case $(\mathrm{Sz}=0 / 2)$, DFT calculation does not converged, which suggested an unstable spin state. Side view of spin density of $\mathrm{Sz}=6 / 2$ is shown in Fig.6(c). It should be noted that even in case of bi-layer graphene-ribbon the highest spin state is more stable, which fortunately suggests a possibility of strong magnetism.

Quadric layer with $\left[\mathrm{C}_{48} \mathrm{H}_{3} \times 4\right]$ super-cell was sown in Fig.8. The most stable spin state is the highest spin state $\mathrm{S}_{z}=12 / 2$. In other lower spin states like $\mathrm{S}_{z}=6 / 2,3 / 2$ and $0 / 2$, DFT calculations did not converged predicting unstable ones. Quadric layer's result suggested drastic increase of magnetization and a capability of three dimensionally layered ribbon.

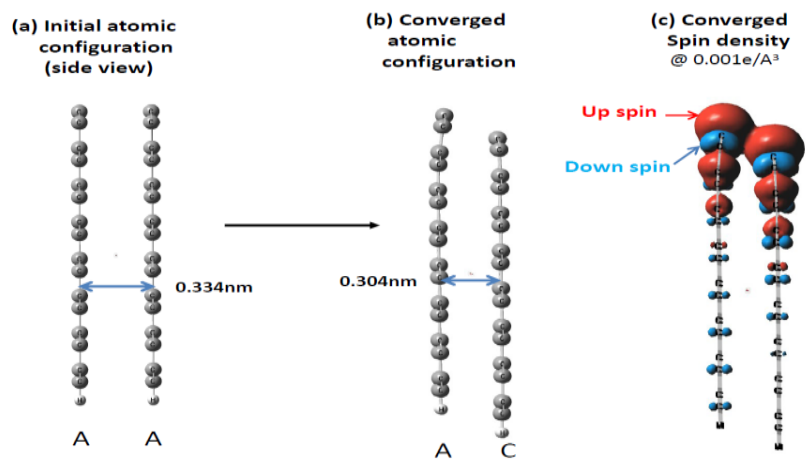

Fig.6 Side view of bilayer graphene ribbon. Initial 
atomic configuration is the AA stack parallel structure as shown in (a). Converged result is illustrated in (b) as the AC stack. Also, converged spin density is shown in (c). Up-spin clouds became close together at bare (radical) carbon edges at upper end in (c).

(a) $\mathrm{Sz}=6 / 2$

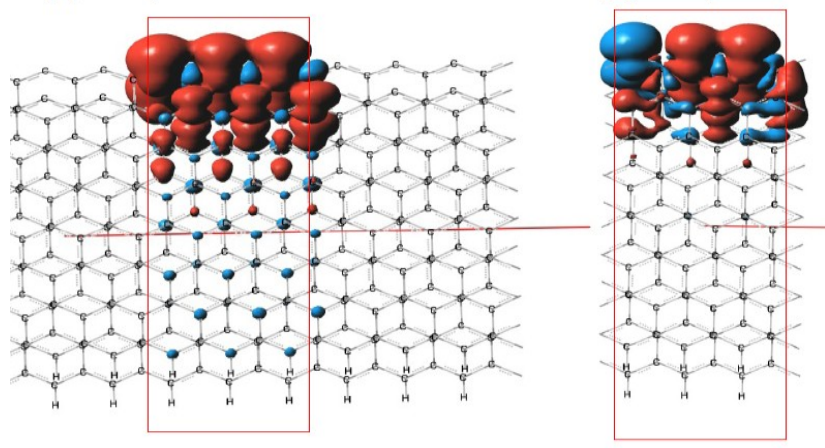

Fig.7 Spin density map of bi-layer graphene-ribbon in a plane view. Total energy of the highest spin state $\mathrm{Sz}=6 / 2$ in (a) is $1.6 \mathrm{kcal} / \mathrm{mol}$ lower and stable than that of $\mathrm{Sz}=2 / 2$ in (b). This suggests a possibility of doubling a saturation magnetization.

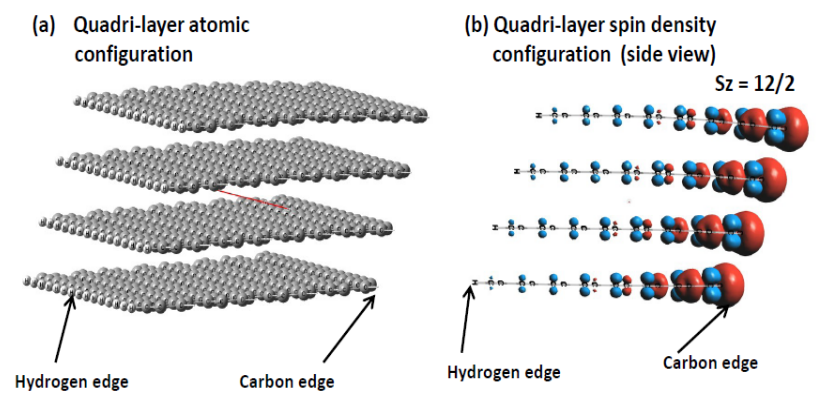

Fig.8 Quadric layer graphene-ribbon atomic configuration bird eye view in (a) and spin density side view in (b). The most stable spin state is the highest spin state $\mathrm{Sz}=12 / 2$. Four times magnetization enhancement was expected.

\section{Conclusion}

Carbon based material is very attractive for realizing a future ultra high density 100 tera-bit/inch ${ }^{2}$ class magnetic information storage media. Especially, graphene-ribbon has a nano-meter width and long straight line looks like a recording track. Here, graphene-ribbon magnetic properties and geometrical optimization were analyzed based on the first principles density functional theory. Multiple spin state analysis was done on typical model with bare (radical) carbons on one side zigzag edge, whereas mono hydrogenated on another side. Optimizing atomic configuration, self-consistent calculation demonstrated that a total energy of the highest spin state was the most stable one than that of lower spin states, which suggested a possibility of strong magnetism. In order to increase areal magnetization density, bi-layer and quadric layer model were analyzed, which resulted that also the highest spin state is the most stable one. Increasing layer numbers is an effective way to enhance strong magnetism.

\section{References}

1)Y. Shiroishi, F. Fukuda, I. Tagawa, H. Iwasaki, S.Takenoiri, H. Tanaka, M. Mutoh and N. Yoshikawa : IEEE Trans. on Mag., 45, 3816 (2009)

2) Y. Shiroishi: Magune, 5, (2010), Review in Japanese

3) C.F. Hirjibehedin, C-Y. Lin, A.F. Otte, M. Ternes, C. P. Lutz, B. A. Jones and A. J. Heinrich： Science, 317, 1199 (2007)

4) P. Esquinazi, D. Spemann, R. Hohne, A. Setzer, K. Han, and T. Butz: Phys. Rev. Lett., 91, 227201(2003)

5) K. Kamishima, T. Noda, F. Kadonome, K. Kakizaki and

N. Hiratsuka: ,J. of Mag. and Magn. Mat., 310 e346 (2007)

6) T. Saito, D. Nishio-Hamane, S. Yoshii, and T. Nojima: Appl. Phys. Lett., 98, 052506 (2011)

7) Y. Wang, Y. Huang, Y. Song, X. Zhang, Y. Ma, J. Liang and Y. Chen: Nano Letters ,9, 220(2009)

8) J. Cervenka, M. Katsnelson and C. Flipse: Nature Physics online Oct. 04 (2009)

9) H. Ohldag, P. Esquinazi, E. Arenholz, D. Spemann, M.

Rothermal, A. Setzer, and T.Butz: New Journal of Physics, 12, 123012 (2010)

10) K. Kusakabe and M. Maruyama: Phys. Rev. B, 67, 092406(2003)

11) M. Maruyama and K. Kusakabe: J. Phys. Soc. of Japan, .73, 656 (2004)

12) N. Ota, N. Gorjizadeh and Y. Kawazoe: J. Magn. Soc. Jpn., 34, 573 (2010)

13) N. Ota, N. Gorjizadeh and Y. Kawazoe: J. Magn. Soc. Jpn., 35, 360 (2011)

14) N. Ota, N. Gorjizadeh and Y. Kawazoe: J.Magn.Soc.Jpn., 36, 36 (2012)

15) P. Hohenberg and W. Kohn: Phys. Rev., 136, B864 (1964)

16) W. Kohn and L. Sham: Phys. Rev., 140, A1133(1965)

17) J. P. Perdew, K. Burke and M. Ernzerhof: Phy. Rev. Lett., 77, 3865(1996)

18) M. Frisch, G. Trucks, H. Schlegel et al : Gaussian 03 package software, Gaussian Inc. Wallington CT USA (2009)

19) R. Ditchfield, W. Hehre and J. Pople : J. Chem. Phys., 54,724(1971)

20) Z. Liu, K. Suenaga, P. Harris,and S. Iijima : Phys. Rev. Lett., 102, 015501 (2009)

21) H. Hibino, S. Mizuno, H. Kageshima, M. Nagase, and H. Yamaguchi : Phys. Rev. B 80, 085406 (2009)

22) K. V. Bets and B. I. Yakobson : Nano Res., 2, 161 (2009)

23) P. V. Avramov, D. G. Fedorov, P. B. Sorokin et al : J. Phys. Chem. Lett., 3, 2003 (2012)

Received October 9, 2012; Revised January 28, 2013;

Accepted February 6, 2013 